\title{
Brine percolation and the transport properties of sea ice
}

\author{
K. M. Golden \\ Department of Mathematics, University of Utah, Salt Lake City, UT 84112-0090, U.S.A.
}

\begin{abstract}
Sea ice is distinguished from many other porous composites, such as sandstones or bone, in that its microstructure and bulk material properties can vary dramatically over a small temperature range. For brine-volume fractions below a critical value of about $5 \%$, which corresponds to a critical temperature of about $-5^{\circ} \mathrm{C}$ for salinity of 5 ppt, columnar sea ice is effectively impermeable to fluid transport. For higher brine volumes, the brine phase becomes connected and the sea ice is permeable, allowing transport of brine, sea water, nutrients, biomass and heat through the ice. Over the past several years it has been found that brine transport is fundamental to such processes as sea-ice production through freezing of flooded ice surfaces, the enhancement of thermal and salt fluxes through sea ice, nutrient replenishment for sea-ice algal communities, and to sea-ice remote sensing. Motivated by these observations, recently we have shown how percolation theory can be used to understand the critical behavior of fluid transport in sea ice. We applied a percolation model developed for compressed powders of large polymer particles with much smaller metal particles, which explains the observed behavior of the fluid permeability in the critical temperature regime, as well as Antarctic data on surface flooding and algal growth rates. Moreover, the connectedness properties of the brine phase play a significant role in the microwave signature of sea ice through its effective complex permittivity and surface flooding. Here we review our recent results on brine percolation and its role in understanding the fluid and electromagnetic transport properties of sea ice. We also briefly report on measurements of percolation we made on first-year sea ice during the winter 1999 Mertz Glacier Polynya Experiment.
\end{abstract}

\section{INTRODUGTION}

Sea ice is a complex, composite material consisting of pure ice with brine and air inclusions, whose size and geometry depend on the ice-crystal structure, as well as the temperature and bulk salinity. An interesting feature of sea ice, known to the earliest polar explorers, is its tendency to desalinate with time. Efforts to understand this fundamental process have led to many important discoveries about the microstructure and the dynamics of the brine phase (Weeks and Ackley, 1982). The dominant desalination mechanism has been found to be gravity drainage (Kingery and Goodnow, 1963; Untersteiner, 1968; Cox and Weeks, 1975; Eide and Martin, 1975; Weeks and Ackley, 1982). As an ice sheet grows, its surface rises higher above sea level, producing a pressure head in the interconnected brine system, driving the underlying brine out of the ice (Eide and Martin, 1975; Weeks and Ackley, 1982). In the presence of a temperature profile which increases with depth, an unstable density distribution exists within the brine, producing convective overturning, as well as an exchange between denser brine within the ice and the underlying sea water (Weeks and Ackley, 1982). The input of brine into the upper ocean is important in ice--ocean interactions, through modification of the density of the surface layer, induction of thermohaline convection, and contributing to the formation of bottom water (Carmack, 1986; Eicken, 1992b). The drainage channels which facilitate brine transport have been studied in numerous works (e.g. Lake and Lewis, 1970; Martin, 1974; Eide and Martin, 1975; Niedrauer and Martin, 1979; Cole and Shapiro, 1998). They consist of large, vertical tubular drainage structures attended by smaller tributaries, like a vertically oriented, radially symmetric river system (Weeks and Ackley, 1982).

One of the key findings which has resulted from the study of the desalination process is that for brine-volume fractions $p$ below a critical value $p_{\mathrm{c}} \approx 5 \%$, columnar sea ice is effectively impermeable to fluid transport, whereas for $p$ above $p_{\mathrm{c}}$, brine or sea water can move through the ice. The relation of brine volume to temperature $T$ and salinity $S$ (Frankenstein and Garner, 1967) implies $p_{\mathrm{c}}$ corresponds to a critical temperature $T_{\mathrm{c}} \approx-5^{\circ} \mathrm{C}$ for $S=5 \mathrm{ppt}$; we refer to this critical behavior as the "law of fives". Perhaps its clearest demonstration in early works appears in Cox and Weeks (1975) and Weeks and Ackley (1982), where the rate of change of salinity $\mathrm{d} S / \mathrm{d} t$ due to gravity drainage is plotted against brine volume. For brine volumes below roughly $5 \%, \mathrm{~d} S / \mathrm{d} t$ vanishes. Indeed, Ono and Kasai (1985) found that the downward permeability of thin sea ice decreases by over two orders of magnitude as the surface temperature is lowered, in a small neighborhood of $-5^{\circ} \mathrm{C}$.

Brine transport is fundamental to such processes as sea-ice production through freezing of flooded ice surfaces (Ackley and others, 1995), the enhancement of thermal fluxes through sea ice (Lytle and Ackley, 1996; Trodahl and others, 2000), nutrient replenishment for sea-ice algal communities (Fritsen and others, 1994) and to remote sensing (Hosseinmostafa and others, 1995; Lytle and Golden, 1995; Golden, 1997c). However, until recently the basic transition controlling brine transport has received little attention. Percolation theory (Broadbent and Hammersley, 1957; Stauffer and Aharony, 1992) has been developed to analyze the properties of materials where connectedness of a given component determines the bulk behavior. 
Recently we have shown (Golden and others, 1998c) that it provides a natural framework to understand the critical behavior of sea ice. In particular, we applied a compressed-powder percolation model to sea-ice microstructure that explains the law of fives, the observed behavior (Ono and Kasai, 1985) of the fluid permeability in the critical temperature regime, as well as data on surface flooding collected recently on sea ice in the Weddell Sea and East Antarctic regions.

Other examples of brine percolation and transport include the observation in the Arctic (Hudier and others, 1995) that a snowstorm and its resultant loading can induce a complete upward flushing of the brine network. The freezing of a surface slush layer, with resultant brine drainage, was observed in the Antarctic to induce convection within the ice, whereby rejected dense brine is replaced by nutrient-rich sea water from the upper ocean (Lytle and Ackley, 1996), fueling autumn blooms of algae in second-year ice (Fritsen and others, 1994). During the autumn freeze-up, this process provided about $70 \%$ of the salt flux into the upper ocean and increased the total heat flux through the overlying ice and snow cover. The proliferation and growth of sea-ice organisms is favored by permeable ice which allows nutrient replenishment (Dieckmann and others, 1991; Eicken, 1992a). As yet another example, it was observed in the Arctic that there was about a 20 day time lag between the onset of spring snowmelt and the input of fresh water into the mixed layer (personal communication from M. G. McPhee, 1995; Maykut and McPhee, 1995). Presumably, part of this lag was the time it took for the ice sheet to warm to above the critical temperature to allow drainage out of the ice (personal communication from M. G. McPhee, 1995).

For remote sensing, surface flooding and subsequent freezing can affect microwave backscatter from sea ice (Hosseinmostafa and others, 1995, Lytle and Golden, 1995), and this surface flooding is often controlled by percolation processes. Moreover, the connectedness of the brine inclusions, as well as their volume fraction and geometry, affects the complex permittivity $\epsilon^{*}$ of sea ice, which determines how electromagnetic waves are scattered from and propagate through the ice. We review a series of rigorous bounds on the effective complex permittivity $\epsilon^{*}$ of sea ice, treated as a general two- (or three-)component random medium (Golden, 1995a, 1997c; Sawicz and Golden, 1995; Golden and others, 1998a). Using the bounds, we explore how the connectedness properties of the brine phase, as well as volume fraction and other geometrical characteristics, affect $\epsilon^{*}$. We also briefly discuss our recent work in obtaining geometrical information about the brine phase, such as volume fraction and inclusion separation, through electromagnetic inversion (Cherkaeva and Golden, 1998; Golden and others, 1998b).

Finally, we discuss measurements of percolation in firstyear sea ice in the Mertz Glacier polynya, Antarctica: the areal density of brine tubes extending to the surface during some warm episodes, and the penetration of a staining liquid (beet juice) through the brine structures in extracted sea-ice blocks to better expose their geometrical properties.

\section{PERCOLATION AND THE FLUID PERMEABILITY OF SEA IGE}

Percolation theory (Broadbent and Hammersley, 1957; Grimmett, 1989; Bunde and Havlin, 1991; Stauffer and
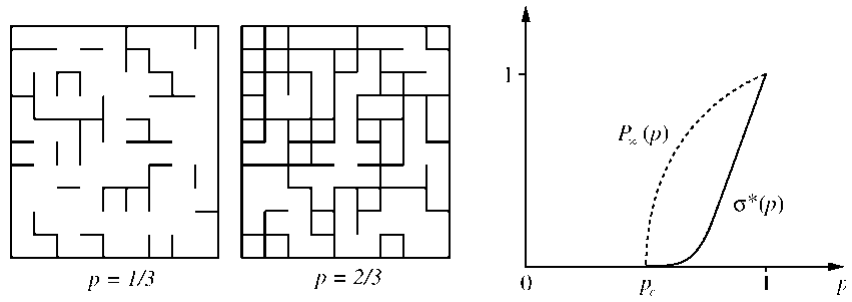

\begin{abstract}
Fig. 1. Typical configurations of the two-dimensional lattice in bond percolation, below $(p=1 / 3)$ and above $(p=2 / 3)$ the percolation threshold $p_{\mathrm{c}}=1 / 2$, and graphs of the infinitecluster density $P_{\infty}(p)$ and effective conductivity $\sigma^{*}(p)$.
\end{abstract}

Aharony, 1992) was initiated by Broadbent and Hammersley (1957) with the introduction of a simple lattice model to study the flow of air through permeable sandstones used in miners' gas masks. In subsequent decades, this theory has been used to successfully model a broad array of disordered materials and processes, including flow in porous media like rocks and soils (Berkowitz and Balberg, 1993; Sahimi, 1995), fractures (Hermann, 1991), doped semiconductors (Shklovskii and Efros, 1984) and various types of disordered conductors (Clerc and others, 1990; Bergman and Stroud, 1992) like piezoresistors, thermistors (Mclachlan and others, 1990), radarabsorbing composites (Priou, 1992), thin metal films (Davis and others, 1991) and polar firn (Shabtaie and Bentley, 1994). The original percolation model and its generalizations have been the subject of intensive theoretical investigations, particularly in the physics (Bunde and Havlin, 1991; Stauffer and Aharony, 1992) and mathematics (Kesten, 1982; Grimmett, 1989) communities. One reason for the broad interest in the percolation model is that it is perhaps the simplest purely probabilistic model which exhibits a type of phase transition.

The simplest form of the lattice percolation model (Stauffer and Aharony, 1992) is defined as follows. Consider the $d$-dimensional integer lattice $\mathbf{Z}^{d}$, and the square (or cubic) network of bonds joining nearest-neighbor lattice sites. To each bond, with probability $p, 0 \leq p \leq 1$, we assign a 1 , meaning it is open, and with probability $1-p$ we assign a 0 , meaning it is closed. Groups of connected open bonds are called open clusters, and the size of a cluster is just the number of open bonds it contains. In the percolation model there is a critical probability $p_{\mathrm{c}}, 0<p_{\mathrm{c}}<1$, called the percolation threshold, at which the average cluster size diverges and an infinite cluster appears, so that the open bonds percolate. In two dimensions $p_{\mathrm{c}}=0.5$, and in three $p_{\mathrm{c}} \approx 0.25$. Typical open-cluster configurations in $d=2$ for $p=1 / 3$ and $p=2 / 3$ are shown in Figure 1 . For $p \geq p_{\mathrm{c}}$, the infinite-cluster density $P_{\infty}(p)$, defined as the probability that the origin (or any point, by translation invariance) is contained in the infinite cluster, or $P_{\infty}(p)=$ $\lim M_{\infty}(L, p) / L^{d}$, where $M_{\infty}(L, p)$ is the mass of the infi$L \overrightarrow{\mathrm{t}}^{\infty}$ cluster contained in a box of side $L$. At the percolation threshold, the infinite cluster has a self-similar, fractal structure, with $M_{\infty}\left(L, p_{\mathrm{c}}\right) \sim L^{d_{\mathrm{f}}}$ as $L \rightarrow \infty$, where $d_{\mathrm{f}} \leq d$ is the fractal dimension. In two dimensions $d_{\mathrm{f}}=91 / 48 \approx 1.9$ is a conjectured exact result, and $d_{\mathrm{f}} \approx 2.5$ in three dimensions (Bunde and Havlin, 1991; Stauffer and Aharony, 1992). The graph of $P_{\infty}(p)$ for $d=2$ is shown in Figure 2. In the neighborhood of $p_{\mathrm{c}}$, with $p \geq p_{\mathrm{c}}, P_{\infty}(p)$ is believed to exhibit the scaling behavior

$$
P_{\infty}(p) \sim\left(p-p_{\mathrm{c}}\right)^{\beta}, \quad p \rightarrow p_{\mathrm{c}}^{+},
$$

where $\beta$ is the percolation critical exponent, which satisfies 




Compressed powder

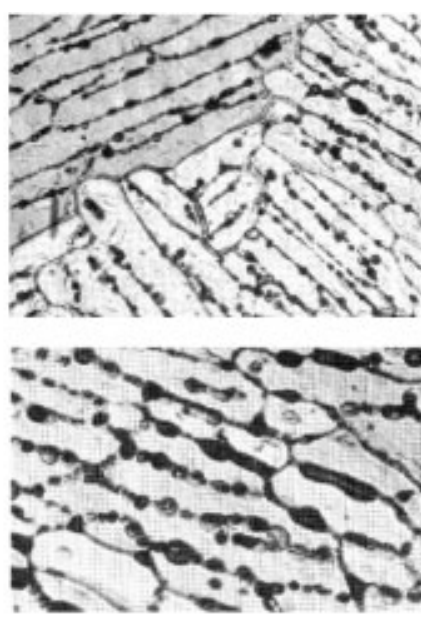

Sea ice a

b

Fig. 2. Comparison of the microstructures of (a) compressed powder of large polymer particles of radius $R_{\mathrm{p}}$ and small metal particles of radius $R_{\mathrm{m}}$ (Malliaris and Turner, 1971), and (b) sea ice (Arcone and others, 1986).

$\beta \leq 1$ (Chayes and Chayes, 1986b), and in $d=2$ it is conjectured that $\beta=5 / 36$ (Bunde and Havlin, 1991).

The percolation model deals only with the geometrical aspects of connectedness in disordered media, yet we are interested in the transport properties as well. Then we consider a random resistor network, where the bonds are assigned the conductivities $\sigma=1$ and $\sigma=h \geq 0$ with probabilities $p$ and $1-p$. With $h=0$, the effective conductivity $\sigma^{*}(p)$, defined via Kirchoff's laws, vanishes for $p<p_{\mathrm{c}}, \sigma^{*}(p)=0$ (for a rigorous formulation of both the lattice and continuum problems see Golden and Papanicolaou, 1983; Golden, 1992; or see section 3 below for a brief treatment of the continuum case). Near the threshold with $p>p_{\mathrm{c}}, \sigma^{*}(p)>0$ is believed to exhibit the power-law behavior

$$
\sigma^{*}(p) \sim\left(p-p_{\mathrm{c}}\right)^{t}, \quad p \rightarrow p_{\mathrm{c}}^{+},
$$

where $t$ is the conductivity critical exponent, with $1 \leq t \leq 2$ in $d=2,3$ (Golden, 1990, 1992, 1997d), and numerical values $t \approx 1.3$ in $d=2$ and $t \approx 2.0$ in $d=3$ (Stauffer and Aharony, 1992). The effective conductivity $\sigma^{*}(p)$ in the $d=2$ lattice case is shown in Figure 1. Analogously we may consider a random pipe network with effective fluid permeability $\kappa^{*}(p)$ exhibiting similar behavior $\kappa^{*}(p) \sim\left(p-p_{\mathrm{c}}\right)^{e}$, where $e$ is the permeability critical exponent, with $e=t$ (Chayes and Chayes, 1986a; Sahimi, 1995; Golden, 1997d). Critical exponents characterizing the behavior of transport near $p_{\mathrm{c}}$ include $t, \delta$ and $\gamma$ defined by

$$
\begin{array}{ll}
\sigma^{*}\left(p_{\mathrm{c}}, h\right) \sim h^{1 / \delta}, & h \rightarrow 0^{+}, \\
\frac{\partial \sigma^{*}}{\partial h} \sim\left(p-p_{\mathrm{c}}\right)^{-\gamma}, & p \rightarrow p_{\mathrm{c}}{ }^{+}, \quad h=0,
\end{array}
$$

and a spectral "gap" exponent $\Delta$. Such exponents, like $t$, are generally believed to exhibit universality, meaning that they depend only on dimension and not on the type of lattice, although continuum models can exhibit non-universal behavior, with exponent values different from the lattice case. For example, consider the Swiss-cheese model in $d=2$, where circular discs (or spheres in $d=3$ ) are removed at random from a uniform medium of unit (electrical or fluid)

conductivity (Kerstein, 1983; Halperin and others, 1985; Feng and others, 1987; Stauffer and Aharony, 1992; Sahimi, 1994). Near the percolation threshold, the transport properties are dominated by flow through the narrow necks between non-overlapping discs (or spheres). Since the widths of such necks can vary throughout the system, there is a distribution of bond conductivities $\sigma$ in an equivalent Voronoi network model (Kerstein, 1983). Moreover, this distribution is singular near $\sigma=0$, which can lead to violations of universality with values of the transport critical exponents in $d=3$ higher than for the lattice case, and $e \neq t$ (Halperin and others, 1985; Feng and others, 1987).

While the critical exponents for continuum systems might differ from their lattice counterparts, we have recently proven that $t, \delta, \gamma$ and $\Delta$ for both lattice and continuum models satisfy the same equations, or scaling relations, and that these equations are the same as those satisfied by the analogous exponents from the theory of phase transitions in statistical mechanics (Golden, 1997a). Our results are based on a direct, analytic connection we have found between transport coefficients for two-component random media in lattice or continuum settings, such as the effective conductivity, permittivity, diffusivity or fluid permeability, and the order parameters in statistical mechanical models, such as the magnetization $M$ of an Ising ferromagnet. This connection establishes the virtual equivalence of a transport transition at a percolation threshold, and a phase transition in statistical mechanics such as the Curie point of a ferromagnet or a liquid/solid transition, through shared Stieltjes integral representations for the coefficients in the two problems, and the introduction of a partition function and free energy associated with a given composite microstructure (Golden, 1995b).

If the above classical lattice percolation model is applied to sea ice, where the open bonds represent brine and the closed bonds represent ice, then $p_{\mathrm{c}}$ would be about $25 \%$ in $d=3$, which is much larger than the observed $5 \%$. Even continuum models, such as ellipsoidal brine inclusions randomly distributed in an ice host, a commonly used model for sea ice, exhibit critical volume fractions in the $20-40 \%$ range (DeBondt and others, 1992). Instead consider the critical behavior of composites made up of conducting particles suspended in an insulating matrix (Mclachlan and others, 1990), and the problem of finding microstructures that reduce $p_{\mathrm{c}}$. For some flexible polymer composites designed to be highly conducting, it was found that by compacting powders of large polymer particles with much smaller metal particles, low critical-volume fractions of the (more expensive) metal particles are required to significantly lower the resistance of the composite (Kusy and Turner, 1971; Malliaris and Turner, 1971). The resulting microstructure of such compressed powders is strikingly similar to the cellular microstructure of columnar sea ice, as shown in Figure 2. The key parameter in predicting the conduction threshold for compressed powders is the ratio $\xi=R_{\mathrm{p}} / R_{\mathrm{m}}$ of the radii of the large polymer particles to the smaller metal particles (Malliaris and Turner, 1971; Kusy, 1977). An approximate formula for the critical-volume fraction for percolation of the small metal spheres in a compressed powder is given by

$$
p_{\mathrm{c}}=\left(1+\frac{\xi \phi}{4 x_{\mathrm{c}}}\right)^{-1}
$$

where $\phi$ is a reciprocal planar packing factor, and $x_{\mathrm{c}}$ is a critical surface-area fraction of the larger particles which must be covered for percolation by the smaller particles 



Fig. 3. Comparison of (a) the electrical conductivity of compressed powders of large polyethylene particles of radius $R_{\mathrm{p}}$ and small nickel particles of radius $R_{\mathrm{m}}$, where $\xi=R_{\mathrm{p}} / R_{\mathrm{m}}=$ 16 (data points from Malliaris and Turner, 1971) and (b) the fluid permeability $\kappa(T)$ of thin young sea ice as a function of surface temperature (data points from Ono and Kasai, 1985). The transport properties of both materials exhibit critical behavior characteristic of a percolation transition. We have also indicated a second transition for $\kappa(T)$ at the melting point $T_{\mathrm{c}}^{\mathrm{melt}}$, where $\log \kappa(T)$ must increase rapidly.

(Kusy, 1977). Values based on microstructural analysis giving good agreement with conductivity experiments are $x_{\mathrm{c}}=0.42$ and $\phi=1.27$ (which we use also as a reasonable approximation for sea ice). An alternative approach to approximating $p_{\mathrm{c}}$ which yields similar quantitative results can be found in Janzen $(1975,1980)$. For large $\xi, p_{\mathrm{c}}$ is not very sensitive to the exact value of $\xi$. For example, a range of $44-17$ in $\xi$ gives only a range of $3-7 \%$ in $p_{\mathrm{c}}$. Using photomicrographs of sea-ice microstructure and typical brine-inclusion sizes (Weeks and Ackley, 1982), we measured the corresponding parameter for sea ice, obtaining an average of $\xi \approx 24$. Applying the compressed-powder percolation model (Kusy, 1977) yields a critical brine volume for columnar sea ice of about $5 \%$. This result will vary with ice-crystal structure. For example, the slightly higher values of $p_{\mathrm{c}}$ (lower $\xi$ ) observed in Ono and Kasai (1985) are caused by the more random distributions of brine inclusions in granular ice as compared to columnar ice. The compressed-powder model explains why sea ice exhibits
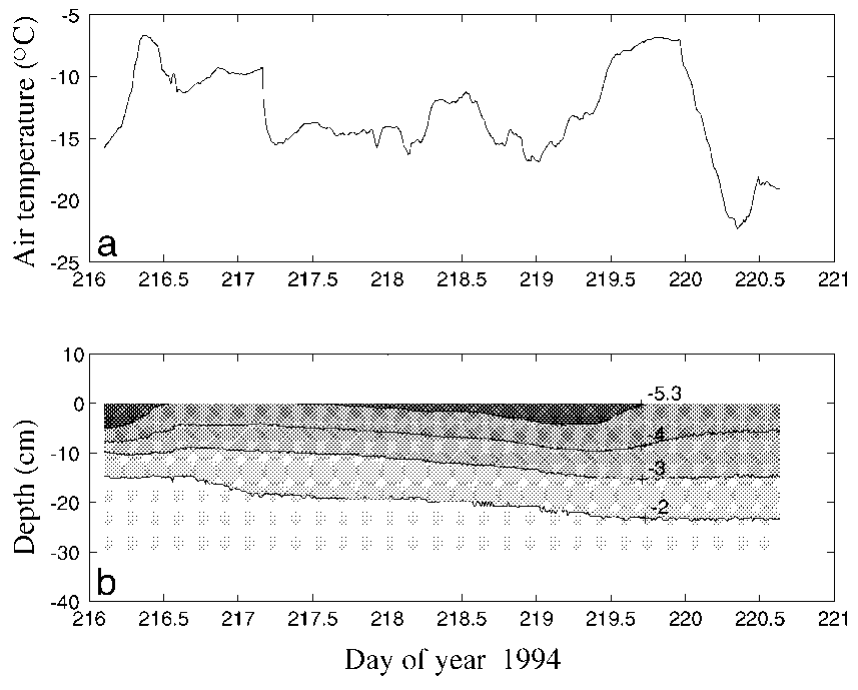

Fig. 4. Temperature contours in sea ice during the Maud Rise drift camp of the ANZFLUX experiment in the eastern Weddell Sea (b), along with air temperatures ( a). The black top layer represents ice which is effectively impermeable to fluid transport, and is not present during warm storms, so that brine may percolate to the surface. Subsequent freezing of the slush layer is an important ice-growth mechanism in the region.

such low values for $p_{\mathrm{c}}$, as compared to the 20-40\% range one might expect, and provides reasonable estimates for $p_{\mathrm{c}}$, which depends only on the geometry of the two phases. Observations show, however, that fluid transport then proceeds primarily through large-diameter brine channels (Weeks and Ackley, 1982; Fritsen and others, 1994; Lytle and Ackley, 1996). Dynamic models will likely be needed for analysis of brinechannel formation beyond the initial onset of percolation at $p_{\mathrm{c}}$, although it is interesting to note the existence of the "independent crossings" of a sample in standard percolation models (Chayes and Chayes, 1986a). They have large separations in comparison to the microstructural (or lattice-spacing) scale for $p$ near $p_{\mathrm{c}}$, on the order of a correlation length (Bunde and Havlin, 1991; Stauffer and Aharony, 1992), and play much the same role for transport as the brine channels.

Comparison of the electrical conductivity of compressed powders and the fluid permeability of sea ice in Figure 3 shows that near the critical temperature, sea-ice permeability displays the same characteristic behavior exhibited in general by transport coefficients of composite media near a percolation threshold (Mclachlan and others, 1990; DeBondt and others, 1992). The data in Figure 3b are thus best fit not with a straight line as in Ono and Kasai (1985) but with an "S" curve that captures the actual behavior of $\kappa(T)$ in the critical regime near the percolation threshold $T_{\mathrm{c}}^{\text {perc }}$.

Compressed powders with low $p_{\mathrm{c}}$ exhibit large, nonuniversal values of $t$ ranging between 2 and 7 (Mclachlan and others, 1990). A rough estimate for the fluid-permeability critical exponent $e$ for sea ice based on the data in Figure $3 \mathrm{~b}$ is about 2.5, although there is significant uncertainty in this estimate (but $e$ is probably 2-4). Given that for lattice models we have shown in $d=3$ that $e=t \leq 2$ (Golden, 1990, 1992), it is likely that sea ice exhibits non-universal behavior, perhaps indicating the importance of the very small necks through which brine must flow near the percolation threshold. Much more experimental work needs to be done to determine the actual range of $e$, how it depends on 
the type of sea ice and other factors, and if indeed the behavior is non-universal.

Data collected on Antarctic sea ice directly demonstrate the significance of the percolation threshold. During the winter ANZFLUX experiment (McPhee and others, 1996) in the eastern Weddell Sea, we encountered a thin ice pack, typically $20-60 \mathrm{~cm}$ thick. Unusually large vertical oceanic heat fluxes resulted in ice basal melt rates of up to $3 \mathrm{~cm} \mathrm{~d}^{-1}$ (average was $1 \mathrm{~cm} \mathrm{~d}^{-1}$ ) which could have melted the ice in a short period. The persistence of the ice depended on flooding of the surface and the subsequent freezing of this slushy snow/brine mixture to form snow ice, which replaced the ice melting on the bottom (Ackley and others, 1995). The surface flooding was controlled by upward brine percolation. Temperature profiles measured hourly during a 5 day drift camp over Maud Rise at about $65^{\circ} \mathrm{S}, 4^{\circ} \mathrm{E}$ (Fig. 4) indicated that while most of the sea-ice layer remained above the critical temperature for percolation, the top $5 \mathrm{~cm}$ or so of the sea ice was impermeable, except during the typically warm storms. Sufficient loading led to surface flooding, which subsequently froze. This cyclic process occurred twice during the 5 day drift. The impermeable layer, defined by temperatures below a critical temperature of about $-5.3^{\circ} \mathrm{C}$, disappeared with the onset of the first storm during day of year 216 (4 August 1994). For about a day the entire ice sheet was permeable, and by noon of the next day we observed a thick layer of slushy snow consisting of $30-50 \%$ liquid brine. Subsequently, a cold period set in, the slush froze and the impermeable cap returned as the frozen slush. Then another storm moved in, with resultant warming, flooding and freezing. Late in the evening of day 219 during a warm storm, we observed large "boils" on the snow surface, which were apparently brine percolating up through the ice.

A similar type of flooding event was observed during the winter HIHO HIHO experiment (Lytle and others, 2000) in the East Antarctic sea-ice pack at about $65^{\circ} \mathrm{S}, 145^{\circ} \mathrm{E}$. The data, which are discussed in Golden and others (1998c), demonstrate clearly that an air-temperature increase alone can cause the permeability phase transition.

As a final example, consider the algae bloom observed in a porous sea-ice layer at depth 10-30 cm during autumn 1992 in the western Weddell Sea (Fritsen and others, 1994). From day of year 60 (29 February) to day 81 standing stocks of pigments in the ice were increasing at a rate of $0.8 \mathrm{mg} \mathrm{m}^{-1} \mathrm{~d}^{-1}$, yet after day 81 the algal growth rate was reduced to onetenth the earlier value. Day 81 is when the downward advancing critical isotherm of $T_{\mathrm{c}} \approx-4^{\circ} \mathrm{C}$ passed through the bottom of the algal layer, effectively cutting off the community from significant nutrient replenishment, as ice above this isotherm was impermeable. The critical temperature higher than $-5^{\circ} \mathrm{C}$ is understood by noting that the ice surrounding the algal layer was granular, and has a higher $p_{\mathrm{c}}$, yet a salinity of only about 5 ppt.

It has been demonstrated that sea ice exhibits a percolation transition at a critical temperature. As mentioned above, recently it has been found that this type of behavior in composite materials is mathematically analogous to a phase transition in statistical mechanics, like water at its freezing point or a ferromagnet at its Curie point (Golden, 1997a). Thus $T_{\mathrm{c}}$ may be viewed as a type of phase-transition point, with sea ice at temperatures between $T_{\mathrm{c}}$ and $-1.8^{\circ} \mathrm{C}$ (the freezing point for sea water) being a hybrid phase between liquid and solid.

\section{PERCOLATION AND THE COMPLEX PERMIT- TIVITY OF SEA ICE}

When electromagnetic waves interact with random media or surfaces, a key parameter determining the nature of the interaction and the types of analysis which can be used is the ratio $\zeta / \lambda$, where $\zeta$ is an appropriate measure of the length scale of variations in the medium or surface, and $\lambda$ is wavelength. For example, at C-band with frequency $f=5.3 \mathrm{GHz}$ and freespace wavelength $\lambda_{0}=5.7 \mathrm{~cm}$, the wavelength $\lambda_{\mathrm{si}}$ in sea ice, typically satisfying $\lambda_{0} / 2<\lambda_{\mathrm{si}}<\lambda_{0}$, is much larger than the sub-millimeter scale of variations in the brine microstructure. In this case, the wave cannot resolve the details of this random inclusion microstructure, and the behavior of the wave is determined primarily by an effective, or homogenized, complex permittivity $\epsilon^{*}$, which is a complicated (tensor) function of the permittivities of the constituents of sea ice and the geometry of the microstructure, as well as frequency. In the quasi-static, or infinite, wavelength limit, a time-independent analysis can be used to analyze complex $\epsilon^{*}$. While scattering from the brine inclusions must be incorporated into $\epsilon^{*}$ over much of the microwave region, it is useful to consider a so-called quasi-static regime, where volume scattering from individual inclusions is relatively small, and the behavior is well approximated with a quasi-static analysis.

Due to the wide variety of possible microstructures and the high dielectric contrast of the components of brine and ice, it is in general quite difficult to accurately predict $\epsilon^{*}$ for sea ice, although many "mixing formulas" for $\epsilon^{*}$ have been proposed and compared with experimental data (Hoekstra and Capillino, 1971; Vant and others, 1978; Golden and Ackley, 1981; Stogryn, 1985; Arcone and others, 1986; Sihvola and Kong, 1988), where typically the sea ice is assumed to consist of a host of pure ice containing ellipsoidal brine and air inclusions. While mixing formulas are certainly useful, their applicability to the full range of microstructures presented by sea ice is limited, and the assumptions under which they are derived are not always satisfied, such as when the brine inclusions percolate. Consequently, we have developed (Golden, 1995a, 1997c; Sawicz and Golden, 1995) a comprehensive series of rigorous bounds on $\epsilon^{*}$ for sea ice, valid in the quasi-static regime, which we briefly describe. The sea ice is assumed to be a two-component composite material of brine with complex permittivity $\epsilon_{1}$, which depends on frequency and temperature (Stogryn and Desargant, 1985), and ice of permittivity $\epsilon_{2}$, which depends weakly on frequency and temperature (Mätzler and Wegmüller, 1987) (which we slightly adjust via the Maxwell-Garnett formula to account for the presence of air (Golden, 1997c; Cherkaeva and Golden, 1998)). Our approach is based on a general, analytic continuation method for obtaining bounds on the effective properties of composites (Bergman, 1980; Milton, 1980; Golden and Papanicolaou, 1983), where $\epsilon^{*}$ is treated as an analytic function of $h=\epsilon_{1} / \epsilon_{2}$, and its properties are exploited to obtain the bounds, which apply to any two-component medium, such as snow or slush, which are also of interest for sea-ice remote sensing. (A full treatment of composites with three or more components is more involved mathematically, requiring analysis of holomorphic functions of several complex variables (Golden and Papanicolaou, 1985; Golden, 1986; Milton, 1987; Milton and Golden, 1990).)

The key step in the method is to obtain the Stieltjes integral 



Fig. 5. Comparison of $4.75 \mathrm{GHz}$ data (circles) on the complex permittivity $\epsilon^{*}$ of sea ice at different temperatures (Arcone and others, 1986) with the bounds $R_{1}$ (outer, dotted), $R_{2}$ (inner, dotted), $R_{1}^{\mathrm{mp}}$ (outer, solid), and $R_{2}^{\mathrm{mp}}$ (inner, solid). $R_{1}$ assumes knowledge of the brine volume, and $R_{2}$ assumes statistical isotropy as well. $R_{1}^{\mathrm{mp}}$ and $R_{2}^{\mathrm{mp}}$ further assume that the sea ice is a matrix-particle composite with the indicated q values corresponding to the geometry in the diagram, where $r_{\mathrm{b}}$ is the radius of a disc containing a brine structure, and $r_{\mathrm{i}}$ is the outer radius of an ice annulus (in $d=2$ ). Note that as the temperature increases, the data move across the region $R_{2}$ and $q$ increases, indicating decreased separation of the brine inclusions. For $T=-2.5^{\circ} C$ the matrixparticle assumption is no longer valid, $q=1$, and $R_{1}^{\mathrm{mp}}$ and $R_{2}^{\mathrm{mp}}$ reduce to $R_{1}$ and $R_{2}$.

representation (Bergman, 1978; Golden and Papanicolaou, 1983)

$$
F(s)=1-\epsilon^{*} / \epsilon_{2}=\int_{0}^{1} \frac{\mathrm{d} \mu(z)}{s-z}, \quad s=\frac{1}{1-\epsilon_{1} / \epsilon_{2}},
$$

where $\mu$ is a positive (spectral) measure on $[0,1]$ containing all the information about the geometry of the composite, which is separated from the parameter information con- tained in $s=1 /(1-h)$. Statistical information about the geometry is input through the moments $\mu_{n}$ of $\mu$, which are related to the correlation functions of the brine phase. For example, $\mu_{0}=p_{1}$, the volume fraction of brine. Bounds on $\epsilon^{*}$, or $F(s)$, are obtained by fixing $s$ in Equation (5), varying over admissible measures $\mu$ (or admissible geometries), such as those that satisfy only $\mu_{0}=p_{1}$, and finding the corresponding range of values of $F(s)$ in the complex plane. If just 
$p_{1}$ is known, we obtain a region $R_{1}$ in the complex $\epsilon^{*}$-plane, in which the complex permittivity of sea ice of that brine volume must lie, regardless of geometry. If the sea-ice microstructure is further assumed to be isotropic within the horizontal plane, we obtain a smaller region $R_{2}$. If we further assume that the sea ice is a matrix-particle composite, where the brine phase is contained in separated inclusions, there is a spectral gap, i.e. the support of $\mu$ in Equation (5) lies in an interval $\left[s_{\mathrm{m}}, s_{\mathrm{M}}\right], 0<s_{\mathrm{m}}<s_{\mathrm{M}}<1$, as observed in fundamental work by Bruno (1991). The further the separation of the inclusions, the smaller the support interval $\left[s_{\mathrm{m}}, s_{\mathrm{M}}\right]$, or the larger the spectral gap, and the tighter the bounds. We obtain regions $R_{1}^{\mathrm{mp}}$ and $R_{2}^{\mathrm{mp}}$ which are significant improvements over $R_{1}$ and $R_{2}$ (Sawicz and Golden, 1995; Golden, 1997c). To compare the matrix-particle bounds with data in Arcone and others (1986), we assume that within the horizontal plane, the brine is contained in separated, circular discs, which allows us to utilize the explicit calculations in Bruno (1991) of $s_{\mathrm{m}}$ and $s_{\mathrm{M}}$. In particular, we consider discs of brine of radius $r_{\mathrm{b}}$ which hold random positions in a host of ice, in such a way that each disc of brine is surrounded by a "corona" of ice, with outer radius $r_{\mathrm{i}}$. Then the minimal separation of brine inclusions is $2\left(r_{\mathrm{i}}-r_{\mathrm{b}}\right)$. Such a medium is called a $q$-material, where $q=r_{\mathrm{b}} / r_{\mathrm{i}}, 0<q<1$. For such a geometry, Bruno (1991) has calculated $s_{\mathrm{m}}=\frac{1}{2}\left(1-q^{2}\right)$ and $s_{\mathrm{M}}=\frac{1}{2}\left(1+q^{2}\right)$. Smaller $q$ values indicate well-separated brine (and presumably cold temperatures), and $q=1$ corresponds to no restriction on the separation, with $s_{\mathrm{m}}=0, s_{\mathrm{M}}=1$, so that $R_{1}^{\mathrm{mp}}$ and $R_{2}^{\mathrm{mp}}$ reduce to $R_{1}$ and $R_{2}$. Examination of photomicrographs of the brine microstructure in the sea-ice samples of Arcone and others (1986) indicates that even when the ice is quite cold, the brine inclusions are quite close, and it is very difficult to estimate appropriate values of $q$. Instead, for a given dataset at a particular temperature, we choose a value of $q$ which best captures the data, and it is always quite close to 1 . Computationally we find that because of the high contrast in the components, the bounds $R_{1}^{\mathrm{mp}}$ and $R_{2}^{\mathrm{mp}}$ are extremely sensitive to small changes in $q$ for $q$ near 1 . By carefully comparing our bounds to data over a wide range of temperatures, we have found that as the temperature increases, i.e. as the percolation threshold $T_{\mathrm{c}}$ is approached and the brine inclusions grow closer, the data sweep across from one side of the region $R_{2}$ to the other (while the regions becomes larger as the brine volume increases), and $q$ increases as well. Once the temperature is above $T_{\mathrm{c}}$, the data require that $q=1$, and the matrixparticle assumption is no longer valid. This fascinating behavior is illustrated in Figure 5, which compares data from samples 84-3 and 84-4 ( $S=3.8 \mathrm{ppt}$ ) in Arcone and others (1986) with the bounds as the temperature is varied over a wide range. We have also applied our matrix-particle bounds, developed for sea ice, to analyze the electromagnetic properties of some insulator/conductor composites arising in smart materials technology (Golden, 1997b).

Finally, we mention our work (Cherkaeva and Golden, 1998; Golden and others, 1998b) in developing a rigorous theory of inverse homogenization, based on inversion of the bounds $R_{1}$ and $R_{2}$, which has produced an accurate algorithm for reconstructing the brine volume of sea ice from measurements of the effective complex permittivity. Furthermore, through more sophisticated mathematical techniques, with C. Orum and E. Cherkaeva, we have been able to invert the matrix-particle bounds $R_{1}^{\mathrm{mp}}$ and $R_{2}^{\mathrm{mp}}$ as well to obtain inverse bounds on the separation parameter $q$ from measurements of $\epsilon^{*}$.

\section{MEASUREMENTS OF PERGOLATION ON FIRST- YEAR SEA ICE}

During the recent Mertz Glacier Polynya Experiment (16 July-7 September 1999) aboard RSV Aurora Australis, we conducted measurements of percolation processes on first-year sea ice in the vicinity of $66^{\circ} \mathrm{S}, 144^{\circ} \mathrm{E}$ with V. I. Lytle, A. P. Worby, R. Massom, I. Allison, A. Rada, M. Paget and A. Roberts. As mentioned above, flooding of a snow layer on the surface of sea ice through upward brine percolation, and subsequent freezing of the slush, is an important mechanism for ice production in some regions of the Antarctic. This process is also essential for supplying nutrients to algal communities growing in the ice. The theory outlined in section 2 gives a reasonable prediction of the critical brine-volume fraction, but more development of the model is needed to understand how macroscopic brine channels or tubes form once the threshold is exceeded, and what determines their separation and properties. Moreover, to assess the level of ice production or nutrient replenishment through percolation processes, it is useful to be able to estimate the vertical fluid permeability of the sea ice, where the bulk of the transport is presumably carried by these larger channels. During a particularly warm period around 12-15 August, where air temperatures reached about $0^{\circ} \mathrm{C}$, and the ice surface temperatures were in the $-4^{\circ}$ to $-5^{\circ} \mathrm{C}$ range, we cleared away the snow and observed extensive arrays of brine tubes at the sea-ice surface, which presumably extended to the bottom. We constructed three "percolation pits", or square meter areas with the snow cleared off, during two of the ice stations. We estimated the number of well-developed brine tubes, measured their diameters, measured temperatures and took photos and video of these structures. The estimates for the number of tubes with about $1 \mathrm{~cm}$ diameters were 60-65, 90-100 and 100-120. Interestingly, in the second pit, over about a $15 \mathrm{~min}$ period after the snow was cleared away we observed that about $2 \mathrm{~cm}$ of brine had flooded the surface of the ice in one corner of the pit. It appears that significant flooding is often associated with "blisters" or "pock-marked" regions of the snow surface (perhaps caused by new or blowing snow accumulating on a very wet surface), and aerial photographs were later taken from a helicopter in order to estimate the density of such regions, which were numerous. While we believe that surface flooding and subsequent freezing is only a minor component of ice production in this polynya, our observations show that it is present, and gave us a more quantitative picture of this process than we had previously.

As a further investigation of brine-channel structure and the percolation process, we extracted with a chain-saw three blocks of sea ice of horizontal dimensions about $25 \mathrm{~cm}$ $\times 75 \mathrm{~cm}$ and thicknesses of $37-66 \mathrm{~cm}$. The ice surface temperatures at these stations were about $-9.4^{\circ}$ and $-7.3^{\circ} \mathrm{C}$. The slabs were turned upside down to expose large brine channels, and bright red, salty beet juice was poured onto the ice. The percolation of the beet juice through the sample was videotaped and photographed. While juice that propagated through the large brine channels made its way far into the samples, much of the liquid stopped after traveling only $10 \mathrm{~cm}$ or so, depending on the sample. This was found later through core analysis to correspond to a structural transition between warm columnar ice where percolation was observed, and colder, granular ice with percolation occurring only in large brine channels. "Thin" sections were chain-sawed off one block to better observe the percolation 
front. These experiments provided a demonstration of how brine moves through sea ice, as well as the difference in the percolation thresholds for columnar and granular ice.

\section{AGKNOWLEDGEMENTS}

The author would like to thank S. F. Ackley and V. I. Lytle for providing much of the data appearing in this paper, as well as for their ideas and close collaboration over the years as this work has developed. The author would also like to thank the crew and participants of the Mertz Glacier Polynya Experiment for their role in obtaining the results in section 4 .

\section{REFERENCES}

Ackley, S. F., V. I. Lytle, G. A. Kuehn, K. M. Golden and M. N. Darling. 1995. Sea-ice measurements during ANZFLUX. Antarct. 7. U.S., 30(5), Review 1995, 133-135.

Arcone, S. A., A. J. Gow and S. McGrew. 1986. Structure and dielectric properties at 4.8 and $9.5 \mathrm{GHz}$ of saline ice. 7. Geophys. Res., $91(\mathrm{C12})$, $14,281-14,303$

Bergman, D. J. 1978. The dielectric constant of a composite material - a problem in classical physics. Phys. Rep., Ser. C, 43(9), 377-407.

Bergman, D. J. 1980. Exactly solvable microscopic geometries and rigorous bounds for the complex dielectric constant of a two-component composite material. Phys. Rev. Lett., 44, 1285.

Bergman, D. J. and D. Stroud. 1992. Physical properties of macroscopically inhomogeneous media. Solid State Physics, 46, 147-269.

Berkowitz, B. and I. Balberg. 1993. Percolation theory and its application to groundwater hydrology. Water Resour. Res., 29, 775-794.

Broadbent, S. R. and J. M. Hammersley. 1957. Percolation processes I. Crystals and mazes. Proc. Cambridge Philos. Soc., 53, 629-641.

Bruno, O. 1991. The effective conductivity of strongly heterogeneous composites. Proc. R. Soc. London, Ser. A, 433, 353-381.

Bunde, A. and S. Havlin, eds. 1991. Fractals and disordered systems. New York, Springer-Verlag.

Carmack, E. C. 1986. Circulation and mixing in ice-covered waters. In Untersteiner, N., ed. Geophysics of sea ice. London, etc., Plenum Press, 641-712. (NATO ASI Series B: Physics 146.)

Chayes, J.T. and L. Chayes. 1986a. Bulk transport properties and exponent inequalities for random resistor and flow networks. Comm. Math. Phys, 105, 133-152.

Chayes, J.T. and L. Chayes. 1986b. An inequality for the infinite cluster density in percolation. Phys. Rev. Lett., 56, 1619-1622.

Cherkaeva, E. and K. M. Golden. 1998. Inverse bounds for microstructural parameters of composite media derived from complex permittivity measurements. Waves in Random Media, 8(4), 437-450.

Clerc, J. P., G. Giraud, J. M. Laugier and J. M. Luck. 1990. The electrical conductivity of binary disordered systems, percolation clusters, fractals and related models. Adv. Phys., 39(3), 191-309.

Cole, D. M. and L. H. Shapiro. 1998. Observations of brine drainage networks and microstructure of first-year sea ice. F. Geophys. Res., $103(\mathrm{Cl} 10)$, 21,739-21,750.

Cox, G. F. N. and W. F. Weeks. 1975. Brine drainage and initial salt entrapment in sodium chloride ice. CRREL Res. Rep. 345.

Davis, C. A., D. R. McKenzie and R. C. McPhedran. 1991. Optical properties and microstructure of thin silver films. Optics Comm., 85(1), 70-82.

DeBondt, S., L. Froyen and A. Deruyttere. 1992. Electrical conductivity of composites: a percolation approach. f. Mater. Sci., 27, 1983-1988.

Dieckmann, G. S., M. A. Lange, S. F. Ackley and J. C. Jennings, Jr. 1991. The nutrient status in sea ice of the Weddell Sea during winter: effects of sea ice texture and algae. Polar Biol., 11 (7), 449-456.

Eicken, H. 1992a. The role of sea ice in structuring Antarctic ecosystems. Polar Biol., 12(1), 2-13.

Eicken, H. 1992b. Salinity profiles of Antarctic sea ice: field data and model results. 7. Geophys. Res., $97(\mathrm{Cl0}), 15,545-15,557$.

Eide, L. I. and S. Martin. 1975. The formation of brine drainage features in young sea ice. F. Glaciol., 14(70), 137-154.

Feng, S., B. I. Halperin and P. N. Sen. 1987. Transport properties of continuum systems near the percolation threshold. Phys. Rev. Lett., 35, 197-214.

Frankenstein, G. and R. Garner. 1967. Equations for determining the brine volume of sea ice from $-0.5^{\circ} \mathrm{C}$ to $-22.9^{\circ} \mathrm{C}$. F. Glaciol., 6(48), 943-944.

Fritsen, C. H., V. I. Lytle, S. F. Ackley and C.W. Sullivan. 1994. Autumn bloom of Antarctic pack-ice algae. Science, 266(5186), 782-784.

Golden, K. 1986. Bounds on the complex permittivity of a multicomponent material. 7. Mech. Phys. Solids, 34(4), 333-358.

Golden, K. 1990. Convexity and exponent inequalities for conduction near percolation. Phys. Rev. Lett., 65(24), 2923-2926.

Golden, K. 1992. Exponent inequalities for the bulk conductivity of a hierarchical model. Comm. Math. Phys., 43(3), 467-499.

Golden, K. M. 1995a. Bounds on the complex permittivity of sea ice. F. Geophys. Res., 100 (C7), 13,699-13,711.

Golden, K. M. 1995b. Statistical mechanics of conducting phase transitions. 7. Math. Phys., 36(10), 5627-5642.

Golden, K. M. 1997a. Critical behavior of transport in lattice and continuum percolation models. Phys. Rev. Lett., 78(20), 3935-3938.

Golden, K. M. 1997b. Critical behavior of transport in percolation-controlled smart composites. In Varadan, V. V. and J. Chandra, eds. Mathematics and control in smart structures. Bellingham,WA, International Society for Optical Engineering, 571-581. (SPIE Proceedings 3039.)

Golden, K. M. 1997c. The interaction of microwaves with sea ice. In Papanicolaou, G. and J. Chandra, eds. Wave propagation in complex media. Berlin, etc., Springer-Verlag, 75-94. (IMA Volumes in Mathematics and its Applications 96.)

Golden, K. M. 1997d. Percolation models for porous media. In Hornung, U., ed. Homogenization and porous media. Berlin, etc, Springer-Verlag, 27-43.

Golden, K. M. and S. F. Ackley. 1981. Modeling of anisotropic electromagnetic reflection from sea ice. 7. Geophys. Res., 86(C9), 8107-8116.

Golden, K. and G. Papanicolaou. 1983. Bounds for effective parameters of heterogeneous media by analytic continuation. Comm. Math. Phys., 90, 473-491.

Golden, K. and G. Papanicolaou. 1985. Bounds for effective parameters of multicomponent media by analytic continuation. f. Stat. Phys., 40(5-6), 655-667.

Golden, K. M. and 9 others. 1998a. Forward electromagnetic scattering models for sea ice. IEEE Trans. Geosci. Remote Sensing, GE-36(5), 1655-1674.

Golden, K. M. and 16 others. 1998b. Inverse electromagnetic scattering models for sea ice. IEEE Trans. Geosci. Remote Sensing, GE-36(5), 1675-1704.

Golden, K. M., S. F. Ackley and V. I. Lytle. 1998c. The percolation phase transition in sea ice. Science, 282(5397), 2238-2241.

Grimmett, G. 1989. Percolation. New York, Springer-Verlag.

Halperin, B. I., S. Feng and P. N. Sen. 1985. Differences between lattice and continuum percolation transport exponents. Phys. Rev. Lett., 54(22), 2391-2394.

Herrmann, H. J. 1991. Fractures. In Bunde, A. and S. Havlin, eds. Fractals and disordered systems. New York, Springer-Verlag, 172-205.

Hoekstra, P. and P. Capillino. 1971. Dielectric properties of sea and sodium chloride ice at UHF and microwave frequencies. 7. Geophys. Res., 76 (20), 4922-4931.

Hosseinmostafa, A. R., V. I. Lytle, K. C. Jezek, S. P. Gogineni, S. F. Ackley and R. K. Moore. 1995. Comparison of radar backscatter from Antarctic and Arctic sea ice. 7. Electromagn. Waves Appl., 9(3), 421-438.

Hudier, E. J.-J., R. G. Ingram and K. Shirasawa. 1995. Upward flushing of sea water through first year sea ice. Atmosphere-Ocean, 33(3), 569-580.

Janzen, J. 1975. On the critical conductive filler loading in antistatic composites. 7. Appl. Phys., 46(2), 966-969.

Janzen, J. 1980. Short derivation of the influence of particle size ratio on the conductivity threshold in binary aggregates. F. Appl. Phys., 51 (4), 2279-2280.

Kerstein, A. R. 1983. Equivalence of the void percolation problem for overlapping spheres and a network problem. F. Phys. A, 16, 3071-3075.

Kesten, H. 1982. Percolation theory for mathematicians. Boston, MA, Birkhäuser.

Kingery, W. D. and W. H. Goodnow. 1963. Brine migration in salt ice. In Kingery, W. D., ed. Ice and snow: properties, processes, and applications. Cambridge, MA, M.I.T. Press, 237-247.

Kusy, R. P. 1977. Influence of particle size ratio on the continuity of aggregates. f. Appl. Phys., 48(12), 5301-5303.

Kusy, R. P. and D. T. Turner. 1971. Electrical resistivity of a polymeric insulator containing segregated metallic particles. Nature, 229, 58.

Lake, R. A. and E. L. Lewis. 1970. Salt rejection by sea ice during growth. 7 . Geophys. Res., $75(3), 583-597$.

Lytle, V. I. and S. F. Ackley. 1996. Heat flux through sea ice in the western Weddell Sea: convective and conductive transfer processes. 7. Geophys. Res., 101(C4), 8853-8868.

Lytle, V. I. and K. M. Golden. 1995. Microwave backscatter measurements from first-year pack ice in the eastern Weddell Sea. Antarct. F. U.S., 30 (5), Review 1995, 125-127.

Lytle, V. I., R. Massom, N. Bindoff, A. Worby and I. Allison. 2000. The Wintertime heat flux to the underside of east Antarctic pack ice. F. Geophys. Res., 105 (C12), 28,759-28,769.

Malliaris, A. and D. T. Turner. 1971. Influence of particle size on the electrical resistivity of compacted mixtures of polymeric and metallic powders. $\mathcal{F}$. Appl. Phys., 42(2), 614-618.

Martin, S. 1974. Ice stalactites: comparison of a laminar flow theory with experiment. F. Fluid Mech., 63, Part 1, 51-79. 
Mätzler, C. and U. Wegmüller. 1987. Dielectric properties of fresh-water ice at microwave frequencies. F. Phys. D, 20(12), 1623-1630.

Maykut, G. A. and M. G. McPhee. 1995. Solar heating of the Arctic mixed layer. F. Geophys. Res., $100(\mathrm{C1} 2), 24,691-24,703$.

Mclachlan, D. S., M. Blaszkiewicz and R. E. Newnham. 1990. Electrical resistivity of composites. F. Am. Ceramic Soc., 73(8), 2187.

McPhee, M. G. and 8 others. 1996. The Antarctic Zone Flux Experiment. Bull. Am. Meteorol. Soc., 77(6), 1221-1232.

Milton, G.W. 1980. Bounds on the complex dielectric constant of a composite material. Appl. Phys. Lett., 37, 300-302.

Milton, G.W. 1987. Multicomponent composites, electrical networks and new types of continued fractions I, II. Comm. Math. Phys., 111, 281-327, 329-372.

Milton, G.W. and K. Golden. 1990. Representations for the conductivity functions of multicomponent composites. Comm. Pure. Appl. Math., 43, 647.

Niedrauer, T. M. and S. Martin. 1979. An experimental study of brine drainage and convection in young sea ice. F. Geophys. Res., 84(C3), 1176-1186.

Ono, N. and T. Kasai. 1985. Surface layer salinity of young sea ice. Ann. Glaciol., 6, 298-299.

Priou, A., ed. 1992. Dielectric properties of heterogeneous materials. New York, Elsevier.

Sahimi, M. 1994. Applications of percolation theory. London, Taylor and Francis Ltd.

Sahimi, M. 1995. Flow and transport in porous media and fractured rock. Weinheim, $\mathrm{VCH}$
Sawicz, R. and K. Golden. 1995. Bounds on the complex permittivity of matrix-particle composites. F. Appl. Phys., 78(12), 7240-7246.

Shabtaie, S. and C. R. Bentley. 1994. Unified theory of electrical conduction in firn and ice: site percolation and conduction in snow and firn. 7. Geophys. Res., 99 (B10), 19,757-19,769.

Shklovskii, B. I. and A. L. Efros. 1984. Electronic properties of doped semiconductors. Berlin, Springer-Verlag.

Sihvola, A. H. and J. A. Kong. 1988. Effective permittivity of dielectric mixtures. IEEE Trans. Geosci. Remote Sensing, GE-26(4), 420-429.

Stauffer, D. and A. Aharony. 1992. Introduction to percolation theory. Second edition. London, Taylor and Francis Ltd.

Stogryn, A. 1987. An analysis of the tensor dielectric constant of sea ice at microwave frequencies. IEEE Trans. Geosci. Remote Sensing, GE-25(2), 147-157.

Stogryn, A. and G. J. Desargant. 1985. The dielectric properties of brine in sea ice at microwave frequencies. IEEE Trans. Antennas Propag., AP-33(5), 523-532.

Trodahl, H. J. and 6 others. 2000. Heat transport in McMurdo Sound firstyear fast ice. 7. Geophys. Res., $\mathbf{1 0 5}$ (C5), 11,347-11,358.

Untersteiner, N. 1968. Natural desalination and equilibrium salinity profile of perennial sea ice. 7. Geophys. Res., 73, 1251-1257.

Vant, M. R., R. O. Ramseier and V. Makios. 1978. The complex-dielectric constant of sea ice at frequencies in the range $0.1-40 \mathrm{GHz}$. F. Appl. Phys., 49(3), Part 1, 1264-1280.

Weeks, W. F. and S. F. Ackley. 1982. The growth, structure, and properties of sea ice. CRREL Monogr. 82-1. 